\title{
1. Global spatial sensitivity of runoff to subsurface permeability using the active subspace method
}

$4{ }^{a}$ Hydrologic Science and Engineering Program, Integrated GroundWater Modeling Center, Department of Geology and Geological Engineering, Colorado School of Mines, Golden, CO 80401

${ }^{b}$ Department of Applied Mathematics and Statistics, Colorado School of Mines, Golden, CO 80401

\section{Abstract}

Hillslope scale runoff is generated as a result of interacting factors that include water influx rate, surface and subsurface properties, and antecedent saturation. Heterogeneity of these factors affects the existence and characteristics of runoff. This heterogeneity becomes an increasingly relevant consideration as hydrologic models are extended and employed to capture greater detail in runoff generating processes. We investigate the impact of one type of heterogeneity - subsurface permeability - on runoff using the integrated hydrologic model ParFlow. Specifically, we examine the sensitivity of runoff to variation in three-dimensional subsurface permeability fields for scenarios dominated by either Hortonian or Dunnian runoff mechanisms. Ten thousand statistically consistent subsurface permeability fields are parameterized using a truncated Karhunen-Loéve (KL) series and used as inputs to 48-hour simulations of integrated surface-subsurface flow in an idealized 'tilted-v' domain. Coefficients of the spatial modes of the KL permeability fields provide parameter space for analysis using the active subspace method. The analysis shows that for Dunnian-dominated runoff conditions the cumulative runoff volume is sensitive primarily to the first spatial mode, corresponding to permeability values in the center of the three-dimensional model domain. In the Hortonian case, runoff volume is sensitive to multiple smaller-scale spatial modes and the locus of that sensitivity is in the near-surface zone upslope from the domain outlet. Variation in runoff volume resulting from random heterogeneity configurations can be expressed as an approximately univariate function of the active variable, a weighted combination of spatial parameterization coefficients computed through the active subspace method. However, this relationship between the active variable and runoff volume is more well-defined for Dunnian runoff than for the Hortonian scenario.

8 Keywords: runoff; heterogeneity; permeability; active subspaces; hydrologic model;

9 sensitivity

\footnotetext{
* Corresponding author

Email addresses: jagilber@mymail.mines.edu ( James Gilbert), jejeffer@mymail.mines.edu (Jennifer Jefferson), paul.constantine@mines.edu (Paul G. Constantine), rmaxwell@mines.edu (Reed Maxwell)
} 


\section{Introduction}

The generation and movement of overland flow (or runoff) is a key hydrologic process that drives associated phenomena like streamflow, erosion, and contaminant transport. Local-scale mechanistic explanations for runoff generation have been examined widely in the literature, with the works of Horton (Horton, 1933) and Dunne (Dunne and Black, 1970) defining the framework common to many modern runoff studies. Saturation excess overland flow (Dunne and Black, 1970) occurs when pore space within the soil column is filled with water (saturated) such that addition of water, commonly as lateral subsurface flux, forces water to exfiltrate at the land surface. In contrast, infiltration excess overland flow (Horton, 1933) occurs when the precipitation rate exceeds the ability of the soil surface to absorb and transmit the incoming water. Both processes can lead to local runoff generation at different times or locations in a watershed. Whether runoff generated in one location translates to runoff in a downslope location depends on the state of the soil surface in the intervening flow path. For example, if the flow path is dominated by saturated soils or has very low infiltration capacity, the upslope runoff is more likely to propagate downhill. The realization of runoff at a downslope point on a hillslope or in a watershed is the result of the integrated effects of moisture and hydraulic properties along each surface flow path. This, then, implies that the runoff response may contain some information on the heterogeneity of such properties.

The impacts of land surface property heterogeneity on runoff have been studied extensively - a host of field and simulation studies have helped establish links between near-surface hydraulic conductivity heterogeneity and spatial, temporal, and statistical properties of runoff (e.g. Freeze, 1980; Loague, 1988; Singh, 1997). The sensitivity of runoff to this heterogeneity, however, depends on other, often transitory, factors like prevailing runoff mechanisms or the nature of a precipitation event. Results from Loague (1988) suggest that the degree and resolution of heterogeneity may matter more for some aspects of runoff than others and that Dunnian mechanisms may be less sensitive to hydraulic conductivity variability than runoff arising from Hortonian conditions. Similarly, the results of Smith and Hebbert (1979), Séguis et al. (2002), and Maxwell and Kollet (2008) support the idea that runoff sensitivity to soil property heterogeneity is inversely proportional to rainfall rate for Hortonian conditions - that is, characteristics of runoff depend increasingly on the degree of permeability heterogeneity as rainfall intensity decreases. Work by Woolhiser et al. (1996), Michaelides and Wilson (2007), Maxwell and Kollet (2008), and Meyerhoff et al. (2014) suggests that the spatial arrangement or trend in heterogeneous properties is an important component of the overall effect of heterogeneity. For example, a stochastically generated permeability field with connected regions of low permeability would yield higher runoff than statistically equivalent realizations lacking such random spatial coherence. Maxwell and Kollet (2008) point out, however, that a simple counting of connected low permeability zones alone cannot be used to fully predict the resulting runoff.

While much of the existing literature focuses on runoff simulated assuming a one or two-dimensional representation of subsurface flow, recent studies have begun to examine complexities of runoff response in simulations that incorporate three-dimensional variably saturated subsurface flow. Maxwell and Kollet (2008) demonstrated the complicated interplay among spatial variance in hydraulic conductivity, rainfall rates, and ergodicity for Hortonian overland flow on a hillslope using the integrated hydrologic code ParFlow. 
Building on previous studies indicating that runoff sensitivity to hydraulic conductivity heterogeneity depends on precipitation rate, Smith and Hebbert (1979), Séguis et al. (2002), and Maxwell and Kollet (2008) found that effective runoff behavior may only be possible under limited high rainfall, low variance conditions. Subsequent studies using ParFlow suggest that, while heterogeneity becomes less important for controlling runoff under high water table conditions (i.e. hillslope runoff becomes a function of mean permeability alone), variance in the saturated hydraulic conductivity field controls the partitioning of total runoff outflow between overland flow and base flow (Meyerhoff and Maxwell, 2011).

\section{Methods}

In this study we investigate the sensitivity of accumulated runoff volume, our scalar output of interest, to changes in the spatially varying subsurface permeability field using active subspaces and an integrated hydrologic model, ParFlow. The methods used for this work are described here in six parts: (1) the active subspace method, (2) configuration of the ParFlow hydrologic model, (3) parameterization of the subsurface permeability field, (4) computation of spatial basis functions, (5) development of a global spatial sensitivity metric, and (6) setup of hydrologic scenarios.

\subsection{Active subspaces}

We describe active subspaces for a generic scalar-valued function of several variables, which we denote $f(\mathbf{x})$. In this particular application, $f$ represents the total cumulative runoff volume, and $\mathbf{x} \in \mathbb{R}^{m}$ represents the $m$ parameters of the permeability field; we discuss the permeability parameterization in Sections 2.3 and 2.4. We assume $f(\mathbf{x})$ is differentiable, and we denote the gradient - oriented as a column $m$-vector - by $\nabla f(\mathbf{x})$.

The active subspace from Constantine (2015) is defined by the first few eigenvectors of the following symmetric, positive semidefinite matrix,

$$
\boldsymbol{C}=\int \nabla f \nabla f^{T} \rho d \mathbf{x}=\boldsymbol{W} \Lambda \boldsymbol{W}^{T}
$$

where $\rho=\rho(\mathbf{x})$ is a non-negative weight function on the space of parameters normalized so that $\int \rho d \mathbf{x}=1$. The weight function is given by the model; it is not a parameter of the method. Loosely speaking, $\rho$ quantifies the probability of sets of permeability parameters. The eigenvectors of $\boldsymbol{C}$ depend on $\rho$. If the modeller changes $\rho$, then the analysis must be repeated.

The $\boldsymbol{W}$ in (1) is the $m \times m$ orthogonal matrix of eigenvectors; $\Lambda$ is the diagonal matrix of non-negative eigenvalues in decreasing order. These satisfy the following relationship with $f$ :

$$
\lambda_{i}=\int\left(\nabla f^{T} \mathbf{w}_{i}\right)^{2} \rho d \mathbf{x}, i=1, \ldots, m .
$$

In words, the eigenvalue measures the mean-squared directional derivative of $f$ along the corresponding eigenvector. If the first $n<m$ eigenvalues are much larger than the rest, then the corresponding eigenvectors identify a set of important directions in the space of inputs; perturbing the inputs $\mathbf{x}$ along these important directions changes $f$ more, on average, than perturbations orthogonal to the important directions. We exploit this 
relationship to study the sensitivity of runoff to a properly parameterized permeability field.

One way to estimate the eigenpairs in (1) is with Monte Carlo as in Constantine and Gleich (2015). But such an approach requires access to the gradient $\nabla f(\mathbf{x})$ as a subroutine. In many applications - including ours - such a subroutine is not available, so the gradient must be approximated. Finite difference approximations are infeasible due to the cost of evaluating $f(\mathbf{x})$. Approximating a gradient vector takes $m+1$ evaluations of $f$-one for each perturbation of the inputs. And estimating $\boldsymbol{C}$ with Monte Carlo would require several such gradient approximations. To circumvent this difficulty, we employ a method based on the following observation. Assume that $f$ is well-approximated by a linear function of $\mathbf{x}^{1}$,

$$
f(\mathbf{x}) \approx a_{0}+\mathbf{a}^{T} \mathbf{x}, \quad \nabla f(\mathbf{x}) \approx \mathbf{a} .
$$

In this case, $\boldsymbol{C}$ becomes

$$
\boldsymbol{C} \approx \int \mathbf{a} \mathbf{a}^{T} \rho d \mathbf{x}=\mathbf{a} \mathbf{a}^{T}=\mathbf{w} \lambda \mathbf{w}^{T}
$$

where $\lambda=\|\mathbf{a}\|^{2}$ and $\mathbf{w}=\mathbf{a} /\|\mathbf{a}\|$. This observation yields the following heuristic method (Constantine, 2015, Algorithm 1.3).

1. Choose $N=\mathcal{O}(m)$ - e.g., 2-to-10 times the number of components in $\mathbf{x}$.

2. For $i=1, \ldots, N$, draw $\mathbf{x}_{i}$ at random according to the density $\rho(\mathbf{x})$.

3. Compute $f_{i}=f\left(\mathbf{x}_{i}\right)$-i.e., run the simulation for each set of inputs $\mathbf{x}_{i}$.

4. Use least-squares to compute the coefficients of a linear approximation of $f(\mathbf{x})$ from a set of pairs $\left\{\mathbf{x}_{i}, f_{i}\right\}$. Call these coefficients $\hat{\mathbf{a}}$.

5. Let

$$
\hat{\mathbf{w}}=\hat{\mathbf{a}} /\|\hat{\mathbf{a}}\|
$$

be the vector that defines the one-dimensional active subspace.

This heuristic works well if $f$ is a monotonic function of the components of $\mathbf{x}$. However, it can only uncover a one-dimensional active subspace. If the dimension of the active subspace for a given $f$ should be greater than one, then this method will not reveal it. We verify the quality of the one-dimensional active subspace using the sufficient summary plots in Section 3.

The least-squares-based approach is closely related to the ordinary least-squares technique in sufficient dimension reduction (Cook, 1998), which identifies a dimension reduction subspace for a given set of data comprising a regression (i.e., a set of predictors and noise corrupted responses). Our application's context is different, since there is no random noise in the ParFlow simulation; in other words, if the simulation runs twice with the same inputs, it will produce the same output. Hence, the method's interpretation differs. In particular, we can use the active subspace to probe the sensitivity of runoff to the spatially varying permeability.

\footnotetext{
${ }^{1}$ Runoff is not a linear function of permeability parameters. The linear assumption is used only to develop the heuristic for estimating a one-dimensional active subspace.
} 
The vector $\hat{\mathbf{w}}$ can be interpreted as follows. Let $\mathbf{x}$ be a point in the domain of $f$. Define a vector $\mathbf{v}$ that is orthogonal to $\hat{\mathbf{w}}$, i.e., $\mathbf{v}^{T} \hat{\mathbf{w}}=0$. Then for some small $\varepsilon$,

$$
\int(f(\mathbf{x}+\varepsilon \hat{\mathbf{w}})-f(\mathbf{x}))^{2} \rho d \mathbf{x}>\int(f(\mathbf{x}+\varepsilon \mathbf{v})-f(\mathbf{x}))^{2} \rho d \mathbf{x} .
$$

In words, perturbing the inputs along $\hat{\mathbf{w}}$ changes $f$ more, on average, than perturbing $\mathbf{x}$ in some direction orthogonal to $\hat{\mathbf{w}}$. The stipulation on average is essential; it is a consequence of the integration in (6). Note that $\hat{\mathbf{w}}$ is a direction in the space of $\mathbf{x}$. Its elements indicate the contribution to the direction from each element of $\mathbf{x}$, i.e., each coordinate of the input space, which can be used to measure sensitivity of $f$ to the components of $\mathbf{x}$ (Diaz and Constantine, 2015).

\subsection{Hydrologic model}

We use the integrated hydrologic code ParFlow to simulate subsurface and overland flow in an idealized 'tilted-v' domain (Maxwell et al., 2014; Jefferson et al., 2015). The ParFlow code solves the three-dimensional variably-saturated Richards' equation for subsurface flow with an overland flow boundary condition at the land surface (Ashby and Falgout, 1996; Kollet and Maxwell, 2006). Necessary input parameters for such a simulation include three dimensional spatially explicit properties of the subsurface porous medium and two dimensional spatial properties of the the land surface. Subsurface properties include permeability, water retention and relative permeability relationship parameters (i.e. van Genuchten parameters), porosity, and specific storage. Surface slope and Manning's roughness values are required for each surface cell in the domain.

The tilted-v domain used here follows the form used in other studies (Kollet and Maxwell, 2006; Sulis et al., 2010; Maxwell et al., 2014; Jefferson et al., 2015) and consists of two planar hillslopes converging towards a central planar channel that slopes toward the southern edge of the domain. The model domain has lateral dimensions of 300 meters (m) by $300 \mathrm{~m}$ and a vertical thickness of $16 \mathrm{~m}$. We discretize the domain into 30 rows, 30 columns, and 16 layers such that individual cell dimensions are a uniform $10 \mathrm{~m} \times 10$ $\mathrm{m} \times 1 \mathrm{~m}$. The configuration of the domain is shown in Figure 1. Boundary conditions on the domain are no-flow at the lateral and bottom boundaries and overland flow at the domain top. The planar hillslopes are each $140 \mathrm{~m}$ wide and $300 \mathrm{~m}$ long while the central channel is $20 \mathrm{~m}$ wide and $300 \mathrm{~m}$ long. We assign the planar hillslopes a slope of $1 \%$ in the $\mathrm{x}$-direction and all planes (the hillslopes and channel) a $1 \%$ slope in the y-direction. This idealized configuration eliminates potentially confounding effects of terrain variability while still approximating the form of a real-world headwater drainage.

All input parameters are spatially uniform except the permeability. This allowed us to isolate the sensitivity of runoff to changes only in spatial variability of subsurface permeability. The spatially uniform parameters used in the tilted-v model domain are shown in Table 1 . The assignment of spatially varying permeability values is described in the next section.

\subsection{Parameterizing the permeability field}

Our goal is to quantify the sensitivity of the cumulative runoff volume from the tilted$\mathrm{v}$ domain to changes in the spatially varying permeability field using active subspaces. 

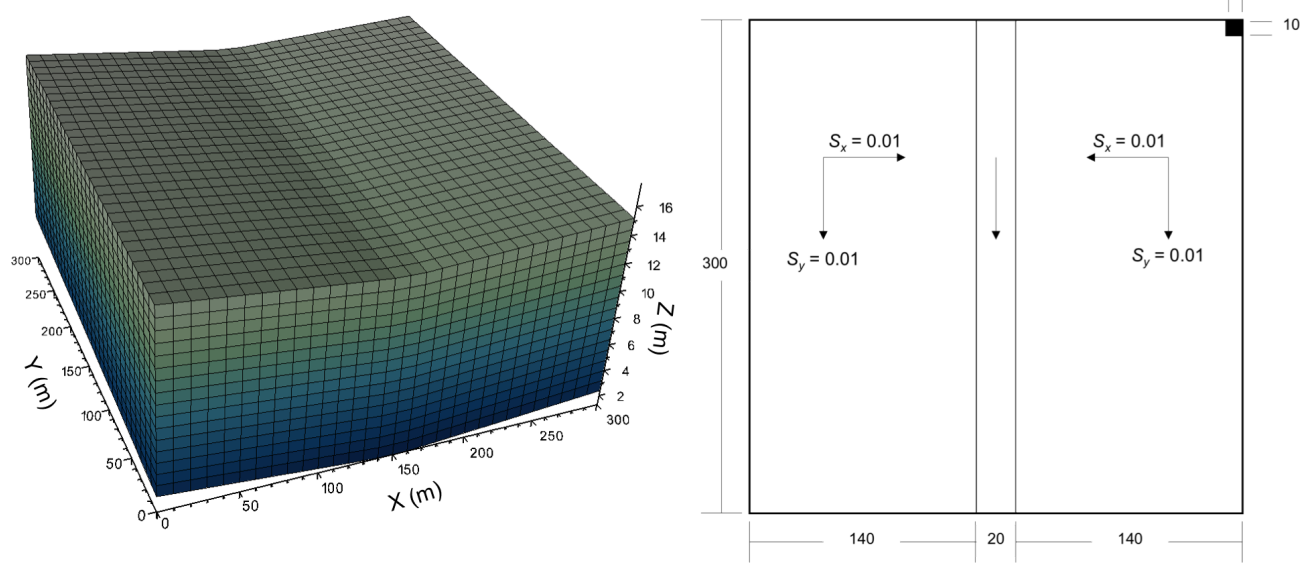

Figure 1: Configuration of the idealized 'tilted-v' domain. (Left) A three dimensional view with a vertical exaggeration of 10. (Right) Plan view, modified from (Jefferson et al., 2015). Domain dimensions are $300 \mathrm{~m} \times 300 \mathrm{~m} \times 16 \mathrm{~m}$.

\begin{tabular}{llr}
\hline Parameter & Value & Units \\
\hline Manning's Roughness & $5.0 \times 10^{-6}$ & $\mathrm{~h} \mathrm{~m}^{-1 / 3}$ \\
Manning's Roughness (channel) & $1.0 \times 10^{-6}$ & $\mathrm{~h} \mathrm{~m}^{-1 / 3}$ \\
Porosity & 0.512 & - \\
Specific Storage & $1.0 \times 10^{-4}$ & $\mathrm{~m}^{-1}$ \\
van Genuchten $\alpha$ & 4.0738 & $\mathrm{~m}^{-1}$ \\
van Genuchten $n$ & 2.0 & - \\
Residual saturation & 0.056 & - \\
\hline
\end{tabular}

Table 1: Spatially uniform subsurface parameters in the ParFlow simulation of the tilted-v domain. 
Recall that $f$ is the cumulative runoff volume, and $\mathbf{x}$ parameterizes the permeability field. In this section we discuss the parameterization.

In principle, any finite dimensional parameterization of the permeability field will suffice - as long as the parameterization and the weight function $\rho(\mathbf{x})$ properly represent the variability one observes in the permeability field. The sensitivity analysis provided by the active subspace is a global analysis. In other words, it measures sensitivity over all possible permeability fields allowed by the chosen parameterization. In contrast, local sensitivity would compute the gradient of runoff with respect to permeability parameters for one permeability field. Global sensitivity metrics enable one to make statements about the runoff over a large collection of permeability fields permitted by the chosen parameterization.

To demonstrate the methodology, we choose a particular parameterization based on a truncated Karhunen-Loéve (KL) series of a Gaussian random field. Such subsurface parameterizations are common in hydrology (Mejia and Rodriguez-Iturbe, 1974; Delhomme, 1979; Clifton and Neuman, 1982; Mantoglou and Wilson, 1982; Tompson et al., 1989; Jaime Gomez-Hernandez and Mohan Srivastava, 1990; Das et al., 2010; Christakos, 2013). First, one assumes a Gaussian random field model of the log permeability field. Gaussian random fields are entirely determined by the mean function $\mu(\mathbf{s})$ and the twopoint spatial correlation function $\mathcal{C}(\mathbf{s}, \mathbf{t})$, where $\mathbf{s}$ and $\mathbf{t}$ are points in the spatial domain. One typically chooses a correlation model and correlation length scale to model perceived smoothness in the permeability; longer correlation lengths yield spatially smoother fields. Assume that the correlation function admits as set of eigenfunction / eigenvalue pairs,

$$
\int \mathcal{C}(\mathbf{s}, \mathbf{t}) \phi_{i}(\mathbf{s}) d \mathbf{s}=\sigma_{i}^{2} \phi_{i}(\mathbf{t}), i=1, \ldots,
$$

where the eigenfunctions are orthonormal,

$$
\int \phi_{i}(\mathbf{s}) \phi_{j}(\mathbf{s}) d \mathbf{s}= \begin{cases}1, & \text { if } i=j \\ 0, & \text { otherwise. }\end{cases}
$$

The truncated KL series approximates the Gaussian random field by a finite series

$$
\log (\kappa(\mathbf{s})) \approx \mu(\mathbf{s})+\sum_{i=1}^{m} \sigma_{i} \phi_{i}(\mathbf{s}) X_{i},
$$

where the $X_{i}$ 's are independent standard Gaussian random variables. The truncation $m$ is typically chosen so that the neglected $\sigma_{i}$ are below some threshold, which determines how much of the field's variance is contained in the truncated approximation.

The random variables $X_{i}$ can be viewed as coefficients of the series expansion. In other words, a realization of $X_{i}$ produces a realization of the log-permeability that is consistent with the modeled spatial correlation function. We treat these coefficients as parameters of the permeability to study spatial sensitivity,

$$
\log (\kappa(\mathbf{s}, \mathbf{x})) \approx \mu(\mathbf{s})+\sum_{i=1}^{m} \sigma_{i} \phi_{i}(\mathbf{s}) x_{i},
$$

where $\mathbf{x}=\left[x_{1}, \ldots, x_{m}\right]$. The subtle difference in notation between (9) and (10) represents an important conceptual difference; the uppercase $X_{i}$ denotes a random variable used to 
model uncertainty in the permeability field, while the lowercase $x_{i}$ denotes a parameter that we can fix and perturb as needed to study the sensitivity of runoff. The $m$-variate Gaussian joint density function for $X_{i}$ is a good choice for $\rho(\mathbf{x})$ in (1). If we choose fixed values of $x_{i}$ that are consistent with the density of $X_{i}$, then the sensitivity analysis is consistent with the modeled uncertainty in the permeability field. In principle, one could choose a different $\rho$ to study sensitivity, but such a choice must be justified.

For computation, the permeability field must be discretized. Assume the solver needs the permeability field at a set of spatial points $\mathbf{s}_{j}$ with $j=1, \ldots, N$, and denote

$$
\kappa_{j}=\kappa\left(\mathbf{s}_{j}, \mathbf{x}\right), \quad \mu_{j}=\mu\left(\mathbf{s}_{j}\right), \quad \phi_{i, j}=\phi_{i}\left(\mathbf{s}_{j}\right) .
$$

Then we can conveniently write the permeability field in vector notation as

$$
\log (\boldsymbol{\kappa})=\boldsymbol{\mu}+\boldsymbol{B} \mathbf{x}
$$

where

$$
\boldsymbol{\kappa}=\left[\begin{array}{c}
\kappa_{1} \\
\vdots \\
\kappa_{N}
\end{array}\right], \quad \boldsymbol{\mu}=\left[\begin{array}{c}
\mu_{1} \\
\vdots \\
\mu_{N}
\end{array}\right], \quad \boldsymbol{B}=\left[\begin{array}{ccc}
\sigma_{1} \phi_{1,1} & \cdots & \sigma_{m} \phi_{m, 1} \\
\vdots & \ddots & \vdots \\
\sigma_{1} \phi_{1, N} & \cdots & \sigma_{m} \phi_{m, N}
\end{array}\right]
$$

We assume the number $N$ of spatial grid points $\mathbf{s}_{j}$ is greater than the number $m$ of parameters $x_{i}$, so $\boldsymbol{B}$ is a tall, thin matrix.

\subsection{Computing the spatial basis}

For this study, we computed the basis $\boldsymbol{B}$ from (12) with a two-step process. First, we used a three-dimensional turning bands algorithm (Tompson et al., 1989) to generate random realizations of permeability fields conforming to model grid dimension with the properties as shown in Table 2. All input variables to the turning bands algorithm were kept constant except an externally generated random seed. The random field was generated using log-transformed permeability values and was not restricted by any internal domain boundaries. The lateral and vertical correlation lengths of $50 \mathrm{~m}$ and $5 \mathrm{~m}$, respectively, were selected from the lower end of the range shown to produce reasonable results using the turning bands method for the given discretization (Tompson et al., 1989).

Second, we obtained spatial basis functions $\boldsymbol{B}$ and a mean permeability value $\boldsymbol{\mu}$ for use with (10) using a principal component analysis applied to 2000 realizations of the turning bands-generated log permeability fields. We retained the first 100 principal components as the spatial basis functions, which was sufficient to ensure that the correlation lengths of the truncated KL representation matched the turning bands representation.

Figure 2 shows plan view and cross-sectional slices for two permeability field realizations; dashed lines on the plan views indicate the three cross-section locations. The permeability realizations shown here correspond to the results of two scenarios tested and discussed in Section 3, with the top images in Figure 2 corresponding to the results Figure 4, the bottom corresponding to results in Figure 5.

\subsection{A global spatial sensitivity metric for runoff}

From (6) and (12), we can construct a spatially varying perturbation of the discretized log permeability field that changes runoff more, on average, than other perturbations. In 

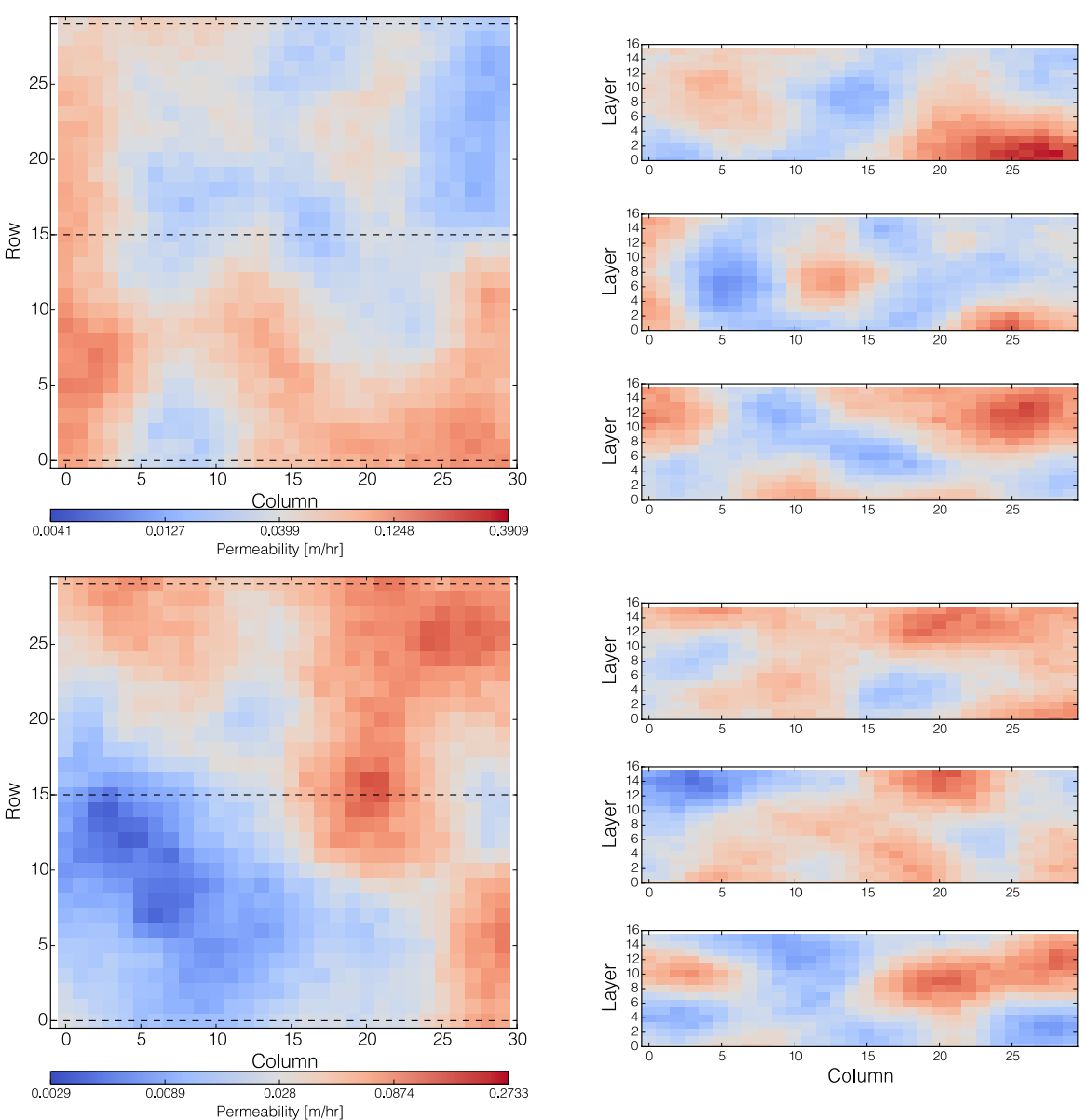

Figure 2: Two three-dimensional permeability field realizations produced through the Karhunen-Loéve method described. Plan views shown on the left, cross-sections on the right. Axis lengths correspond to grid dimensions $(300 \mathrm{~m} \times 300 \mathrm{~m} \times 16 \mathrm{~m})$; column $(\mathrm{dx})$ and row $(\mathrm{dy})$ widths are each $10 \mathrm{~m}$ and layer thickness $(\mathrm{dz})$ is $1.0 \mathrm{~m}$. The three dashed lines on each plan view correspond to the three cross-section locations. The top images correspond to the example results shown for the initially saturated hydrologic scenario. The bottom images correspond to the initially unsaturated hydrologic scenario. 


\begin{tabular}{llr}
\hline Turning bands parameter & Value & Units \\
\hline Geometric mean of permeability & 0.04465 & $\mathrm{~m} \mathrm{~h}^{-1}$ \\
Correlation length, $\lambda_{x}$ & 50.0 & $\mathrm{~m}$ \\
Correlation length, $\lambda_{y}$ & 50.0 & $\mathrm{~m}$ \\
Correlation length, $\lambda_{z}$ & 5.0 & $\mathrm{~m}$ \\
Standard deviation, $\sigma$ & 1.0 & $\mathrm{~m} \mathrm{~h}^{-1}$ \\
Number of lines & 100 & - \\
R zeta & 50.0 & - \\
K max & 100.0 & - \\
$\Delta \mathrm{K}$ & 0.5 & - \\
Seed & randomly generated & - \\
\hline
\end{tabular}

Table 2: Turning bands algorithm input parameter values used to generate random permeability fields that train the spatial bases in the truncated KL series. One hundred terms of the KL were sufficient to represent fields with these statistics.

particular, let $\mathbf{x}$ be a set of coefficients of the parameterized log permeability in (12), and let $\hat{\mathbf{w}}$ be the vector computed in (5). Then for small $\varepsilon$, the runoff with log permeability

$$
\log (\boldsymbol{\kappa})=\boldsymbol{\mu}+\boldsymbol{B}(\mathbf{x}+\varepsilon \hat{\mathbf{w}})=\boldsymbol{\mu}+\boldsymbol{B} \mathbf{x}+\varepsilon \boldsymbol{B} \hat{\mathbf{w}},
$$

deviates more, on average, from runoff with log permeability defined by $\mathbf{x}$ than any permeability whose coefficients $\mathbf{x}$ are perturbed orthgonally to $\hat{\mathbf{w}}$.

The vector $\boldsymbol{B} \hat{\mathbf{w}}$ has the same size as the discretized spatial domain, so we can plot it and interpret its components as a spatially varying field. This vector is our global, spatial sensitivity metric for runoff with respect to log permeability. It is global by construction of $\hat{\mathbf{w}}$ in (5); i.e., it reveals an important one-dimensional perturbation in the space of $\mathbf{x}$ (the $\log$ permeability parameters) that affects $f$ (runoff). And it is spatial because the matrix $\boldsymbol{B}$ transforms a vector in the space of $\log$ permeability parameters $(\mathbf{x})$ to a discretized, spatially varying field.

It's worth emphasizing that the vector $\boldsymbol{B} \hat{\mathbf{w}}$ depends heavily on the parameterization of the permeability field. However, the methodology remains unchanged as long as the parameterization is linear-i.e., any linear combination of spatial basis functions. Such parameterization may or may not be related to the uncertainty modeling in the permeability field. One advantage of the Karhunen-Loéve is the relatively low-dimensional parameterization compared to the number of spatial discretization points. In this application, 14,400 cell values are reduced to 100 parameter values. However, the KarhunenLoéve series validity depends on the ability to (i) model the permeability with a Gaussian random field and (ii) identify the mean and spatial covariance functions.

\subsection{Hydrologic scenarios}

Given that different runoff generating mechanisms suggest varying interactions with the subsurface, we tested the active subspace method using simulated runoff responses generated by imposing uniform saturation excess and infiltration excess runoff mechanisms in the tilted-v ParFlow model. To ensure infiltration excess (Hortonian) overland flow in the simulation we initialized the model with a deep water table (at the bottom of the domain) and defined a 3 -hour, 5 -cm/ $\mathrm{hr}$ precipitation event over model hours one 
through three. We refer to this scenario as initially unsaturated. Runoff simulated at the model outlet under this condition would necessarily result from connected zones of low infiltration capacity. The saturation excess (Dunnian) overland flow generation mechanism was ensured by initializing the domain with a near-surface water table but with no precipitation inputs, similar to the approach used in previous studies (Sulis et al., 2010; Maxwell, 2013). The exclusion of precipitation in this scenario eliminates potentially confounding overland flow generation mechanisms. Thus the simulated runoff outflow in this case is generated as the water table equilibrates and forces downslope exfiltration. We refer to this scenario hereafter as initially saturated. In both scenarios the simulations span 48 hours.

For each scenario, we apply the active subspace heuristic method from Section 2.1 to the 10,000 input/output pairs. These pairs consist of the vector of 100 Gaussian random variables ( $\left\{\mathbf{x}_{i}\right\}$ from Section 2.1 - the inputs) and the total runoff volume $\left(f_{i}=f\left(\mathbf{x}_{i}\right)\right.$ from Section 2.1 - the outputs), respectively.

\section{Results and discussion}

We performed simulations using the two initializations described in Section 2.6. As expected, the initially saturated condition yields an overland flow response that is characterized by an initially high runoff pulse dominated by exfiltration along the hillslopes of the tilted-v. At late time, runoff decreases but is maintained over the subregion where connected high-permeability subsurface zones conduct water forced by local topographic slope and pressure to the surface. The runoff hydrographs in Figure 3a demonstrate this temporal response. The corresponding spatial patterns of runoff, as indicated by surface pressures, for early and late time under initially saturated conditions are shown in Figure 4. The images on the left show variations in positive surface pressure, with darker shades indicating higher pressure (i.e., ponded water depth). Note that the late time pressure field in Figure 4 is the result of a single subsurface permeability realization and that the pattern of surface pressures changes depending on permeability configuration. The contour plots on the right show subsurface pressure in cross-section over the top eight meters of the domain. All contours shown are positive except the one in bold, which indicates the water table. The dashed contour represents pressures that exceed the top of the model domain and are therefore contributing to overland flow.

The plots in Figure 5 show early and late-time pressure fields from one initially unsaturated realization. Runoff responses for this scenario are more varied, with some permeability realizations resulting in zero runoff during the entire 48-hour simulation. The variation in runoff is shown in the outflow hydrograph in Figure 3b: runoff peaks at hour four but varies between zero and approximately 1,300 cubic meters per second. Non-zero runoff for this scenario occurs when a positive surface pressure initiates under the applied precipitation and propagates downslope over low permeability surface zones. The amount of runoff is thus a measure of the connectivity of low-permeability zones at or near the surface. The permeability field corresponding to this particular realization and simulation result is shown in plan view and cross-section in Figure 2. In all realizations however, the applied water wets the vertical profile from the top down, as indicated by the closely-spaced, near-zero negative contours at the top of the cross sections in Figure 5.

The weights vector, $\hat{\mathbf{w}}$ from (5), provides a measure of the global sensitivity of runoff to the 100 input parameters, i.e., the coefficients in the truncated KL representation 

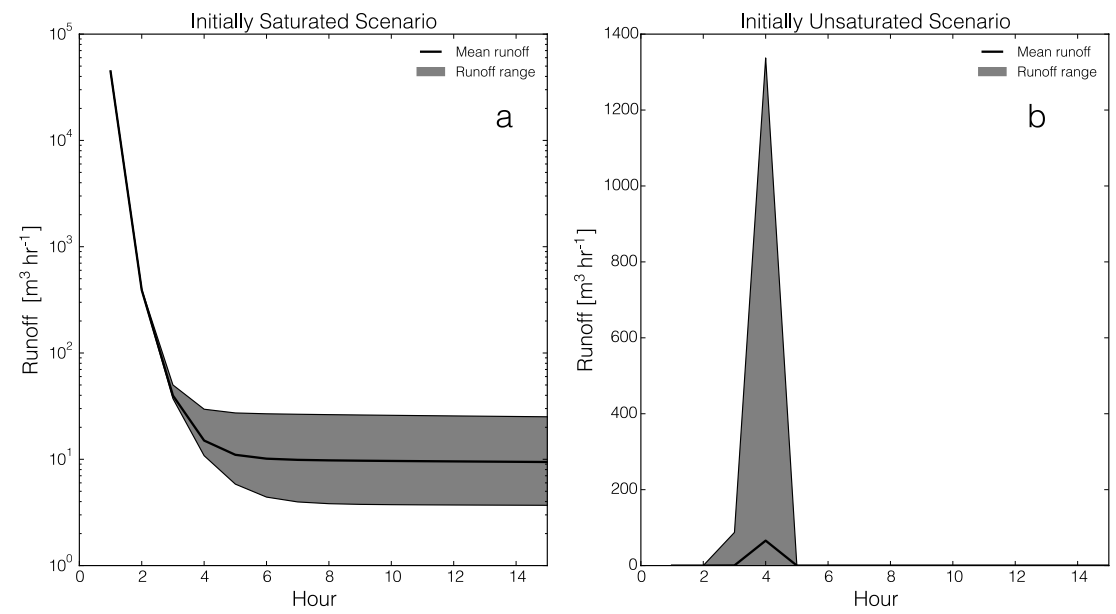

Figure 3: Runoff hydrograph summaries for (a) the initially saturated case and (b) the initially unsaturated case. The black line indicates the mean runoff response. The shaded area marks the range of simulated runoff response. Note logarithmic scale in the initially saturated case (a).

of $\log$ permeability in (12). The weights are shown for the initially saturated case in Figure $6 \mathrm{a}$ and for the initially unsaturated case in Figure 7a. The plots show the spatial sensitivity changes according to the dominant runoff process. For the initially saturated case (Figure 6a), the weight of the first coefficient - corresponding to the first spatial basis function - is nearly one. Furthermore, the weights for the subsequent parameters decrease quickly, with weights for parameters beyond 30 close to zero. Given that the spatial basis functions with lower indices (e.g., 1-30, in this case) correspond to more gradual variability in the permeability field, this result indicates sensitivity of runoff outflow for an initially high water table, and groundwater exfiltration-driven runoff process is controlled by larger scale permeability heterogeneity. In contrast, the weights for the initially unsaturated case do not include a single dominant component and exhibit more spread along increasing parameter indices. While the higher weights for this scenario are still concentrated over the lower range of spatial bases, the difference compared to the initially saturated scenario indicates that Hortonian runoff is more sensitive to smaller scale heterogeneity than for Dunnian runoff. While this finding is not unique (Loague, 1988; Maxwell and Kollet, 2008), the consistency of the result from the active subspace method and previous studies supports the validity of the approach.

We can plot the scalar output (total runoff outflow) as a function the linear combination of the inputs, $\hat{\mathbf{w}}^{T} \mathbf{x}$. We call $\hat{\mathbf{w}}^{T} \mathbf{x}$ the active variable, since changing the scalar $\hat{\mathbf{w}}^{T} \mathbf{x}$ corresponds to moving in $\mathbf{x}$ along $\hat{\mathbf{w}}$. Such plots are called summary plots in the context of regression (Cook, 1998). The summary plot for the initally saturated case is in Figure 6b, and the initially unsaturated case's summary plot is in Figure 7b. We filtered out the results from realizations yielding no runoff such that Figure 7 represents only the non-zero runoff cases. The plots show that total runoff volume from initially unsaturated and initially saturated conditions (Hortonian and Dunnian runoff processes, respectively) are approximately univariate functions of the linear combination of the spa- 

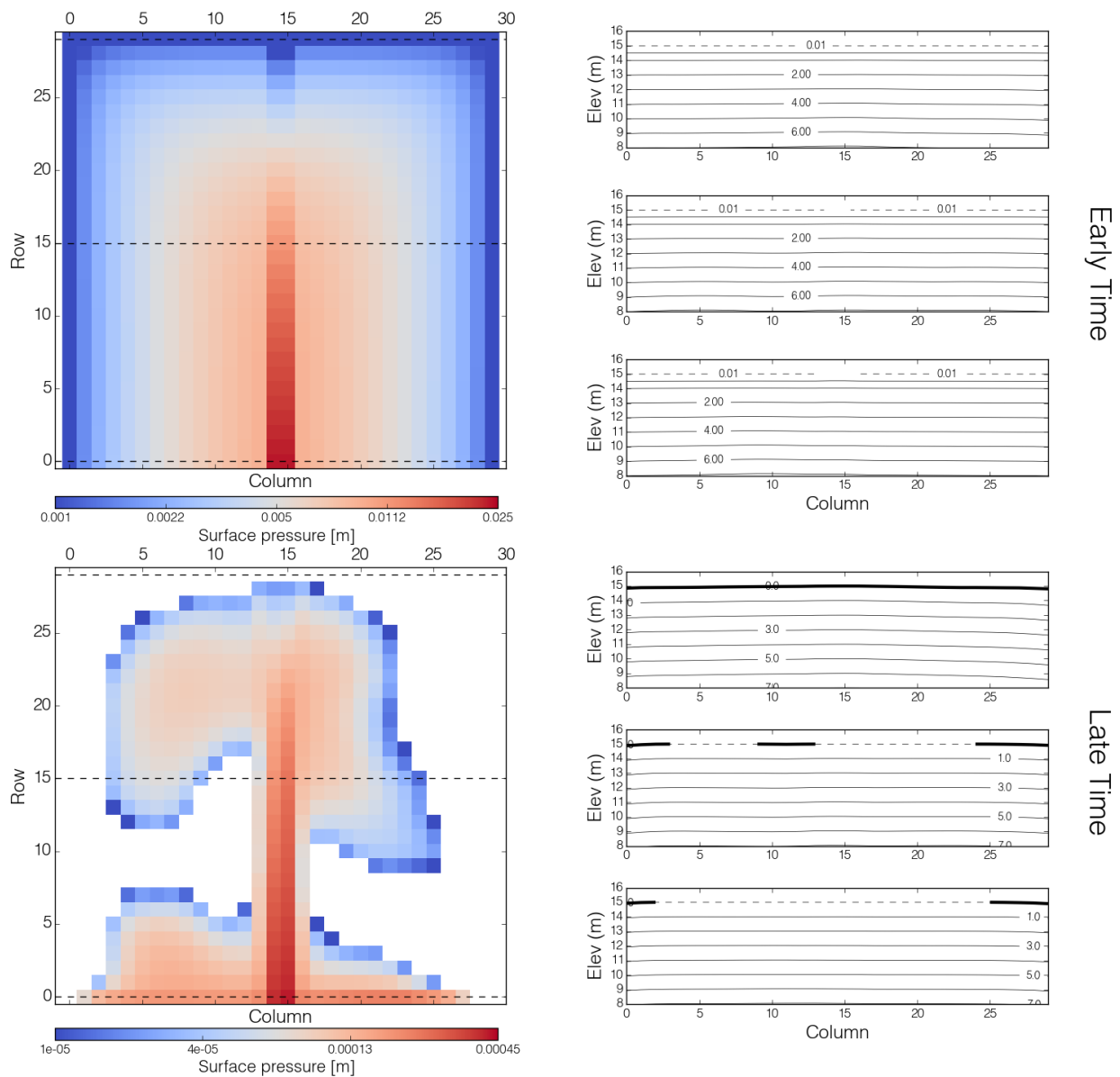

Figure 4: Early and late time pressure fields for the initially saturated case. Plots at left show the pressure field at the model surface. Contour plots at right show selected vertical cross-sections at the north, middle, and south ends of the domain. In the vertical cross-sections, dashed lines represent positive surface pressure and bold lines represent negative surface pressures (unsaturated conditions) The location of cross sections is indicated by dashed lines in plots at left. Axis lengths correspond to model grid dimensions $(300 \mathrm{~m} \times 300 \mathrm{~m} \times 16 \mathrm{~m})$; column $(\mathrm{dx})$ and row (dy) widths are each $10 \mathrm{~m}$ and layer thickness $(\mathrm{dz})$ is $1.0 \mathrm{~m}$. 

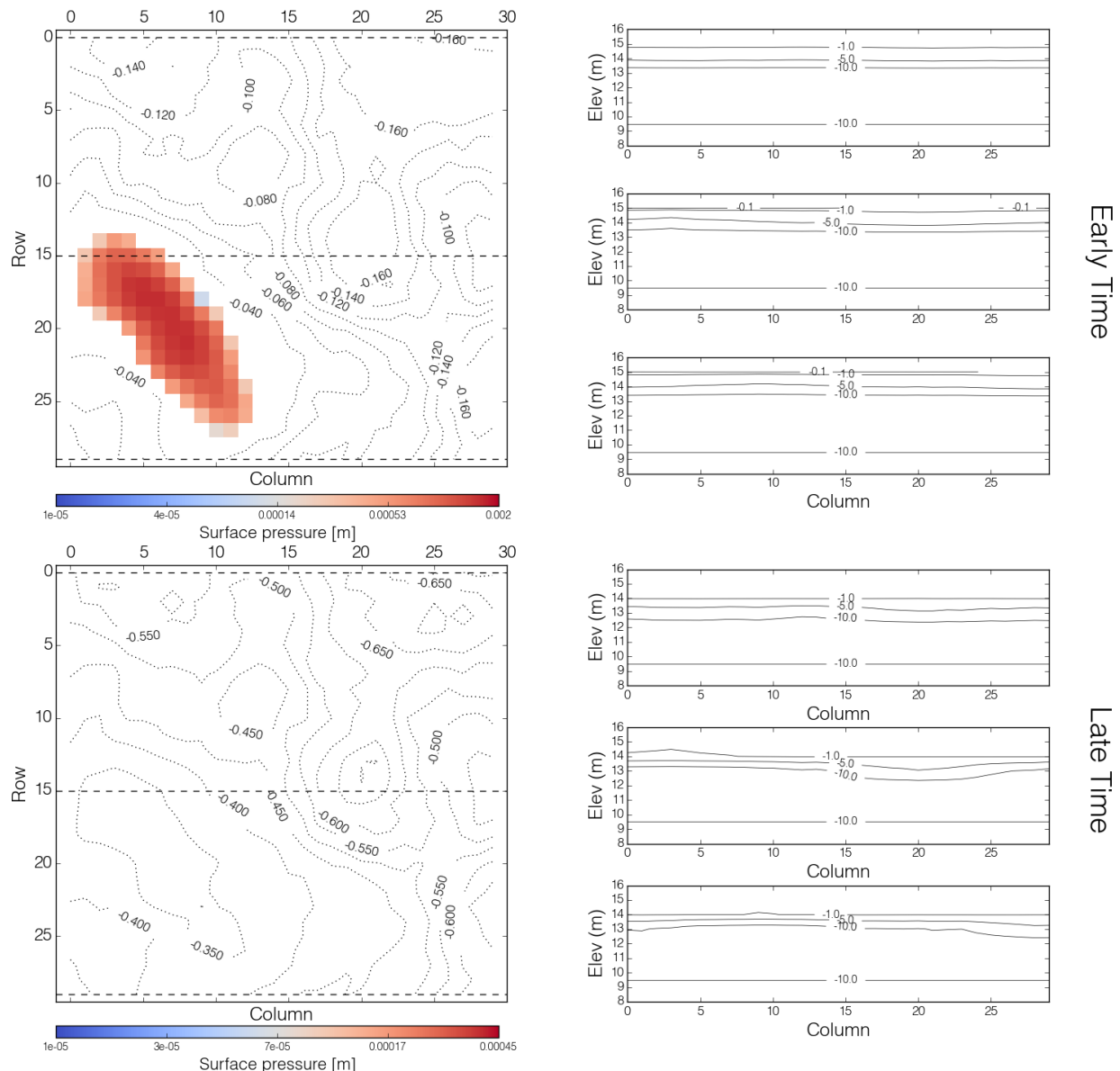

Figure 5: Early and late time pressure fields for the initially unsaturated case. Plots at left show the pressure field at the model surface. Shaded cells indicate positive surface pressure (i.e., ponded water). Dotted contours represent negative pressures associated with unsaturated conditions. Contour plots at right show selected vertical cross-sections at the top, middle, and bottom of the domain. The location of cross sections is indicated by dashed lines in plots at left. Axis lengths correspond to model grid dimensions $(300 \mathrm{~m} \times 300 \mathrm{~m} \times 16 \mathrm{~m})$; column $(\mathrm{dx})$ and row (dy) widths are each $10 \mathrm{~m}$ and layer thickness $(\mathrm{dz})$ is $1.0 \mathrm{~m}$. 
tial basis functions. The mapping of the KL coefficients to the resulting permeability field confounds a straightforward and specific interpretation of the plots. However, the form of the scalar runoff output as a function of the active variable can provide some general insight into the relationship between the permeability field and runoff. In both runoff cases, the cumulative outflow increases directly with increasing active variable. For the initially saturated (Dunne runoff) case, the dominant positive weight means that an increase in the active variable corresponds, primarily, to an increase in permeability of the large-scale (small index) spatial modes. In contrast, the presence of larger negative weights for the initially unsaturated case suggests that an increase in the active variable corresponds to a reduction in the permeability contributed by some of the spatial modes. Therefore, the increase in Hortonian runoff volume with increasing active variable value is a result of a reduction in permeability over certain spatial components. We can surmise something about how and where these components manifest as regions of relative sensitivity, as is discussed in the following paragraphs.

Before we address that aspect of the analysis, however, two key differences between the Dunnian and Hortonian active variable-runoff relationships warrant further examination. First, the nature of the active variable-runoff volume relationship is less welldefined for the Hortonian runoff case (Figure 7). The scatter at low values of the active variable indicates other factors beyond a single linear combination of spatial bases may explain runoff amounts. This suggests greater complexity in the map between the KL coefficients and Hortonian runoff than can be captured by a univariate function of one linear combination of the KL coefficients. However, the apparent relationship shown in the summary plot - though not univariate - suggests the method exposes a useful relationship. Second, although the absolute range in runoff volume is similar in both the Hortonian and Dunnian cases (about $1000 \mathrm{~m}^{3}$ ), the variability of runoff volume relative to the ensemble minimum is different for the Hortonian and and Dunnian cases. To the extent that the variation in runoff volume from near zero to 1000 cubic meters is more profound than variation from a high runoff volume $\left(\sim 45,000 \mathrm{~m}^{3}\right)$ to a higher runoff volume $\left(\sim 46,000 \mathrm{~m}^{3}\right)$, the active variable method suggests a higher sensitivity in the Hortonian case compared to the Dunnian case. This interpretation is consistent with the findings of Meyerhoff and Maxwell (2011) who showed that the effective (geometric mean) hydraulic conductivity of the contributing area is a key control of runoff volume for groundwater exfiltration dominated scenarios. Alternatively, if we consider the active variable as defining an absolute sensitivity (i.e. a unit increase in the active variable corresponds to a certain amount of increased runoff volume), the results of the method suggest comparable overall sensitivities for both cases.

Conforming the vector $\boldsymbol{B} \hat{\mathbf{w}}$ from Section 2.5 to the model grid, we can see the sensitivity of runoff to changes in permeability values on a model grid cell basis. Figure 8 shows this sensitivity for every grid cell, by layer, with the bottom layer (layer 1) in the top left and the top model layer (layer 16) in the bottom right of the figure. The more red the color, the larger the expected change in runoff from any small change in logarithmic permeability at that cell. The plots show that runoff is most sensitive to permeability values in the domain core, with highest values occurring in layers 8-13 and clustered within a ten meter radius of the center of each layer. We interpret this pattern as a consequence of the predominant subsurface flow paths under saturated conditionsas topographic and pressure gradients force water from the outer edges of the domain towards the central channel, much of it must pass through the central core of the domain. 

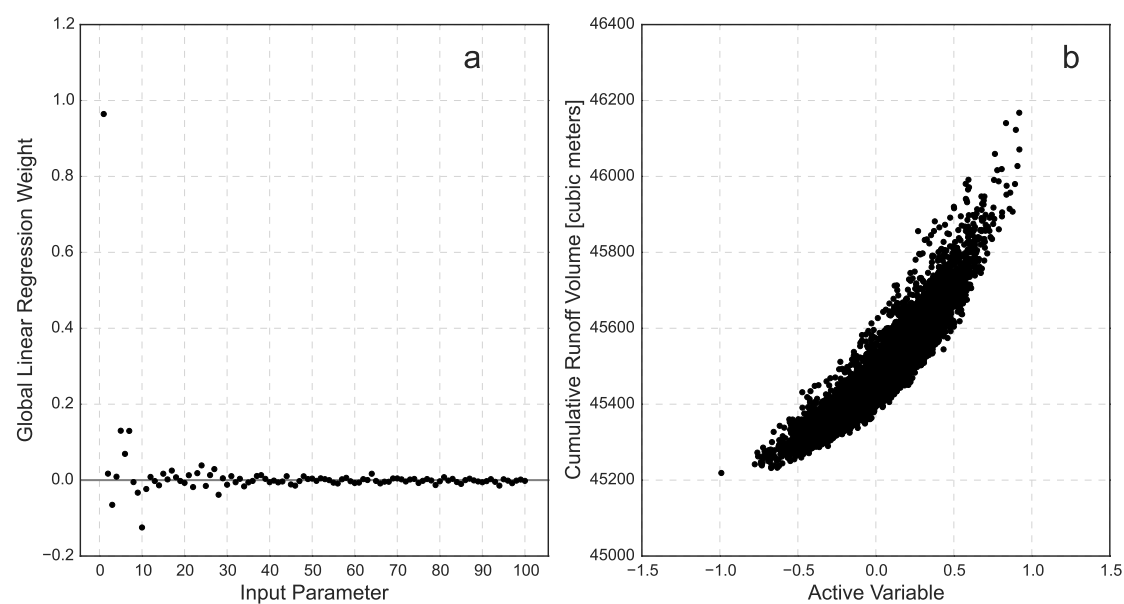

Figure 6: Active variable weights (a) and summary plot (b) for initially saturated simulations. Input parameters represented by the weights plot correspond to coefficients of the spatial basis functions in (12). The active variable is the weighted sum of those coefficients.
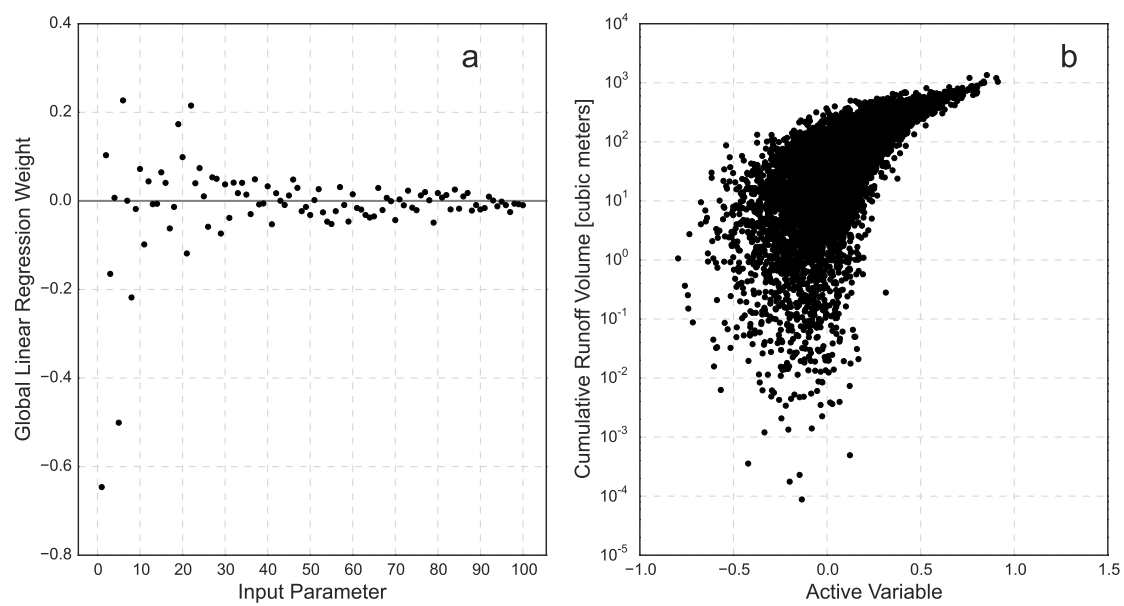

Figure 7: Active variable weights (a) and summary plot (b) for initially unsaturated simulations. Input parameters represented by the weights plot correspond to coefficients of the spatial basis functions in (12). The active variable is the weighted sum of those coefficients. 
Given that the runoff volume analyzed here was accumulated over a finite but transient period, it follows that this volume would depend on the permeability of the zone through which, on average, most flow paths pass.

The sensitivity of Hortonian runoff to permeability perturbations is shown in Figure 9, by layer, for each cell in the domain. The red color again signifies higher sensitivity to change in permeability compared to the blue zones. In contrast to the corresponding plots for the initially saturated scenario, the images in Figure 9 show a broader range of sensitivity, indicating, for example, that changes in permeability in the lowest model layers have comparatively little effect on runoff. Additionally, the zones of highest sensitivity are concentrated in the upper model layers (near the land surface) and along the axis of the tilted-v. One interpretation of the patterns in Figure 9 is that, owing to the initially dry state of the domain, infiltration in the zone of topographic convergence in the $x$ and $y$ directions can significantly alter the amount of runoff leaving the domain, regardless of the precise location in the domain where the runoff was generated initially.

The results in Figures 8 and 9 indicate the active subspace method has potential to provide important insight into runoff processes and other hydrologic phenomena simulated in integrated models. Notably, the method allows a spatially explicit estimation of sensitivity for the entire domain at a simulation cost less than would be required for an enumerative parameter sweep. This capability refines considerably the quantification of changes in spatial sensitivity due to variation in hydrologic process. For example, while previous studies have suggested runoff is more sensitive to local heterogeneity in general under Hortonian conditions, the active subspace and Karhunen-Loéve expansion methods show the locations where that heterogeneity would matter the most.

\section{Summary, conclusions, and future work}

In this study we applied the active subspace method to evaluate the sensitivity of runoff, simulated as the result of variably saturated flow in an idealized three-dimensional domain using the integrated hydrologic code ParFlow, to spatially varying permeability fields with consistent global statistics. The fields were parameterized using the KarhunenLoéve expansion. This allowed representation of a complex, three-dimensional field using a linear combination 100 spatial modes; the coefficients are treated as parameters. We tested two distinct runoff generation scenarios with 10,000 realizations each to assess how sensitivities change under different hydrologic regimes: (i) a Dunne-type saturation excess runoff case imposed by initializing the simulation with a high water table and (ii) a Horton-type infiltration excess runoff case created by raining on an initially dry domain.

The sensitivity of runoff to spatial permeability fields depends on the dominant runoff mechanism. The high weight of the first spatial component, derived from the method as a measure of relative runoff sensitivity to a change in that component, shows that changes in cumulative Dunnian runoff outflow depend almost entirely on large-scale changes in permeability values. In contrast, parameter weights calculated for the Hortonian runoff case show that runoff variation also depends on smaller scale spatial modes, supporting the idea that such runoff is a function of local-scale heterogeneity. Summary plots reveal an approximately univariate relationship between the active variable and runoff volume, with that relationship being more well-defined for the initially saturated case. One potential explanation of the spread in the sufficient summary plots is the presence of a 


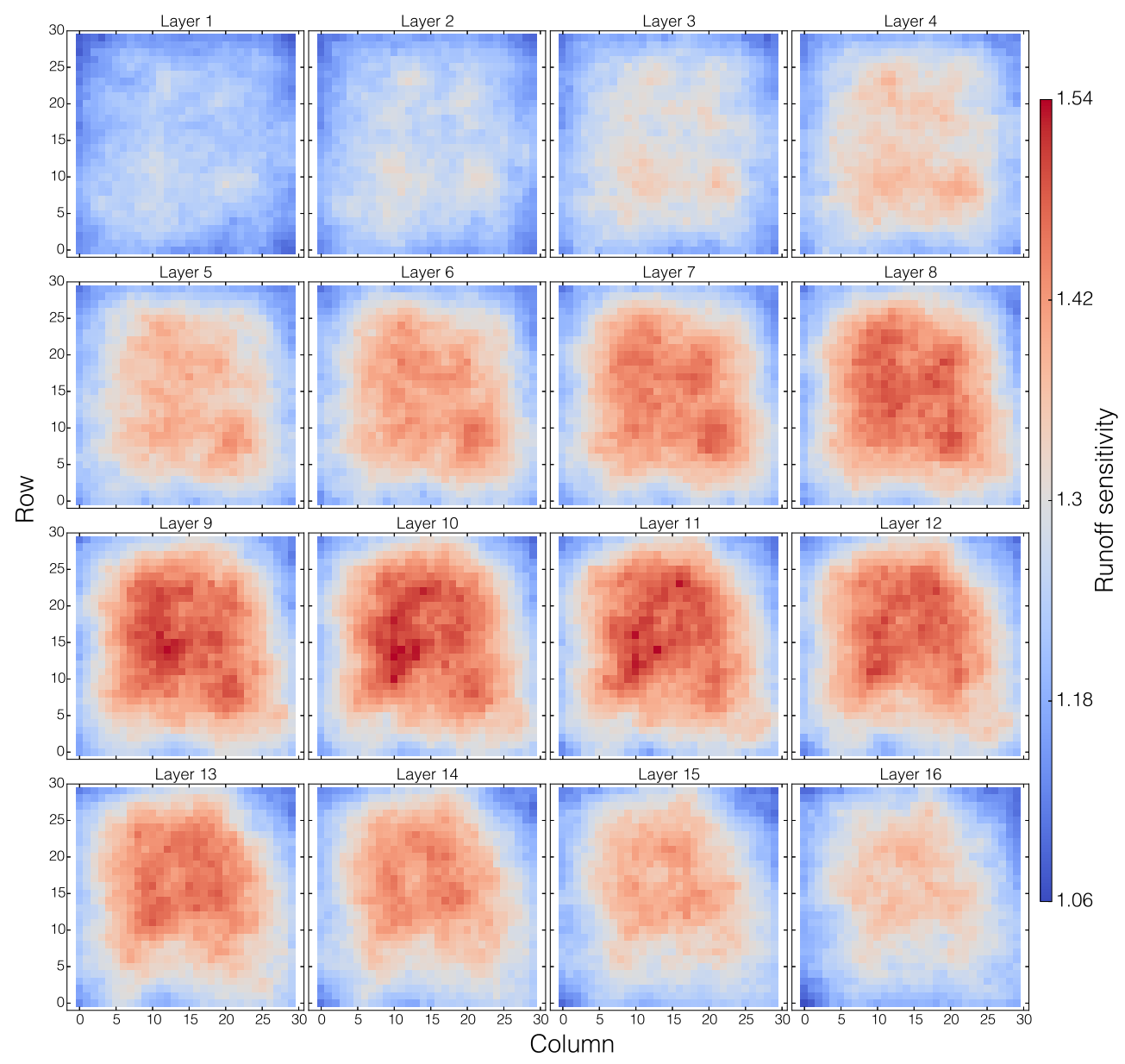

Figure 8: Sensitivity of runoff volume to perturbations in permeability for each cell in the tilted-v model domain for the initially saturated (Dunnian runoff) scenario. Each image shows the sensitivity for a model layer. The bottom model layer (layer 1) is at the top left. The top model layer (layer 16) is at the bottom right. Axis lengths correspond to model grid dimensions ( $300 \mathrm{~m}$ x $300 \mathrm{~m} \mathrm{x} 16 \mathrm{~m}$ ); column $(\mathrm{dx})$ and row (dy) widths are each $10 \mathrm{~m}$ and layer thickness $(\mathrm{dz})$ is $1.0 \mathrm{~m}$. 


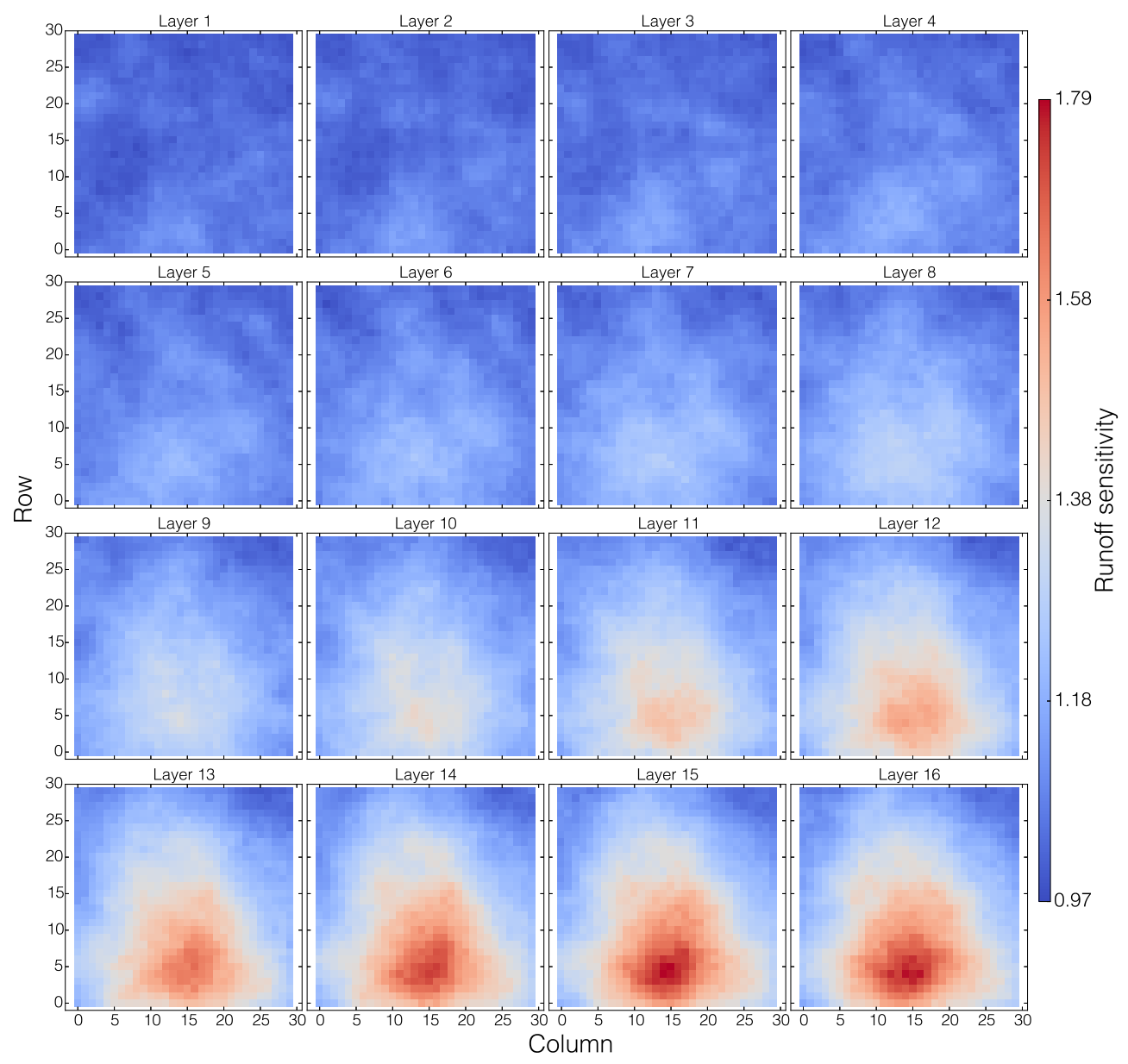

Figure 9: Sensitivity of runoff volume to perturbations in permeability for each cell in the tilted-v model domain for the initially unsaturated (Hortonian runoff) scenario. Each image shows the sensitivity for a model layer. The bottom model layer (layer 1) is at the top left. The top model layer (layer 16) is at the bottom right. Axis lengths correspond to model grid dimensions (300 m x $300 \mathrm{~m} \mathrm{x} 16 \mathrm{~m}$ ); column $(\mathrm{dx})$ and row (dy) widths are each $10 \mathrm{~m}$ and layer thickness $(\mathrm{dz})$ is $1.0 \mathrm{~m}$. 
higher dimensional active subspace not captured by the method based on a global linear model.

Because the global statistics of the random fields were kept constant for all realizations (i.e., the geometric mean, spatial variance and correlation lengths do not change), the sensitivity derived from the method measures the effect of specific permeability patterns on runoff. In other words, it helps quantify the expected change in runoff due to the actual location of heterogeneous values assuming the global spatial statistics are known. For the cases examined, the total variation in cumulative runoff was about $1000 \mathrm{~m}^{3}$. This variation was tied most directly to permeability variations at the central core of the subsurface for the Dunne runoff case and in a localized surface zone near the domain outlet for the Horton runoff case.

The results described here highlight the value of the active subspace method in simplifying the assessment of sensitivity of spatial components of subsurface permeability fields to the complex hydrologic phenomenon of runoff generation and routing. This method holds potential for developing reduced dimension representations of subsurface heterogeneity, guiding and optimizing data collection, and as an additional constraint on the inverse problem. Developing the method towards these uses and applicability to less-idealized situations will require addressing complications excluded from the proof-ofconcept application described in this study. First, runoff mechanisms rarely exist purely in space or time as infiltration excess (Hortonian) or saturation excess (Dunnian) (Loague et al., 2010). The combination of such processes may invalidate the global linearity assumption and will likely warrant a careful assessment of the parameter space and local gradient sampling. Secondly, the idealized cases used here neglected the impact of other atmospheric, topographic, land surface, and subsurface parametric variability on runoff. Given the previously demonstrated dependence of runoff behavior on precipitation intensity (Smith and Hebbert, 1979; Séguis et al., 2002; Maxwell and Kollet, 2008), storm type variation would be an important component to include in future investigations. Such parameters are necessary and uncertain inputs to many hydrologic models. Understanding the sensitivity of simulation results to their variability in the context of permeability variation would help inform, more broadly, the process of integrated hydrologic modeling. The viability of the active subspace method to resolve such relationships in a larger and more process-diverse parameter space remains to be tested. Finally, we tested a method that relied on one parameterization of subsurface heterogeneity that assumed invariant global statistics. The ability of the method to successfully resolve permeability field sensitivities for different random field parameterization schemes and under conditions of spatial non-stationarity warrants further investigation.

\section{Acknowledgments}

This work was supported by funding from the National Science Foundation through its Climate Change, Water and Society (CCWAS) Integrative Graduate Education and Research Traineeship (IGERT) program (http://ccwas.ucdavis.edu/; DGE-1069333). The third author's work is supported by the U.S. Department of Energy Office of Science, Office of Advanced Scientific Computing Research, Applied Mathematics program under Award Number DE-SC-0011077. Computational resources were provided by the Golden Energy Computing Organization at the Colorado School of Mines using resources ac- 
470 quired with financial assistance from the National Science Foundation and the National

471 Renewable Energy Laboratory. 
Ashby, S.F., Falgout, R.D.. A parallel multigrid preconditioned conjugate gradient algorithm for groundwater flow simulations. Nuclear Science and Engineering 1996;124(1):145-159.

Christakos, G.. Random Field Models in Earth Sciences. Elsevier, 2013.

Clifton, P.M., Neuman, S.P.. Effects of kriging and inverse modeling on conditional simulation of the Avra Valley Aquifer in southern Arizona. Water Resources Research 1982;18(4):1215-1234. URL: http://onlinelibrary . wiley.com/doi/10.1029/WR018i004p01215/abstract. doi:10.1029/ WR018i004p01215.

Constantine, P., Gleich, D.. Computing active subspaces with Monte Carlo. arXiv preprint arXiv:14080545v2 2015;

Constantine, P.G.. Active Subspaces: Emerging Ideas in Dimension Reduction for Parameter Studies. SIAM, Philadelphia, 2015.

Cook, R.D.. Regression Graphics: Ideas for Studying Regressions through Graphics. John Wiley \& Sons, Inc., New York, 1998.

Das, N.N., Mohanty, B.P., Efendiev, Y.. Characterization of effective saturated hydraulic conductivity in an agricultural field using Karhunen-Love expansion with the Markov chain Monte Carlo technique. Water Resources Research 2010;46(6):W06521. URL: http://onlinelibrary.wiley.com/doi/10. 1029/2008WR007100/abstract. doi:10.1029/2008WR007100.

Delhomme, J.P.. Spatial variability and uncertainty in groundwater flow parameters: A geostatistical approach. Water Resources Research 1979;15(2):269-280. URL: http://onlinelibrary.wiley.com/ doi/10.1029/WR015i002p00269/abstract. doi:10.1029/WR015i002p00269.

Diaz, P., Constantine, P.G.. Global sensitivity metrics from active subspaces. arXiv preprint arXiv:151004361 2015;.

Dunne, T., Black, R.D.. An Experimental Investigation of Runoff Production in Permeable Soils. Water Resources Research 1970;6(2):478-490. URL: http://onlinelibrary.wiley.com/doi/10. 1029/WR006i002p00478/abstract. doi:10.1029/WR006i002p00478.

Freeze, R.A.. A stochastic-conceptual analysis of rainfall-runoff processes on a hillslope. Water Resources Research 1980;16(2):391-408. URL: http://onlinelibrary.wiley.com/doi/10.1029/ WR016i002p00391/abstract. doi:10.1029/WR016i002p00391.

Horton, R.E.. The Role of infiltration in the hydrologic cycle. Eos, Transactions American Geophysical Union 1933;14(1):446-460. URL: http://onlinelibrary.wiley.com/doi/10.1029/TR014i001p00446/ abstract. doi:10.1029/TR014i001p00446.

Jaime Gomez-Hernandez, J., Mohan Srivastava, R.. ISIM3d: An ANSI-C three-dimensional multiple indicator conditional simulation program. Computers \& Geosciences 1990;16(4):395-440. URL: http: //www.sciencedirect. com/science/article/pii/009830049090010Q. doi:10.1016/0098-3004(90) 90010-Q.

Jefferson, J.L., Gilbert, J.M., Constantine, P.G., Maxwell, R.M.. Active subspaces for sensitivity analysis and dimension reduction of an integrated hydrologic model. Computers \& Geosciences 2015;83:127-138. URL: http://www.sciencedirect.com/science/article/pii/S0098300415300091. doi:10.1016/j.cageo.2015.07.001.

Kollet, S.J., Maxwell, R.M.. Integrated surfacegroundwater flow modeling: A free-surface overland flow boundary condition in a parallel groundwater flow model. Advances in Water Resources 2006;29(7):945-958. URL: http://www.sciencedirect.com/science/article/pii/ S0309170805002101. doi:10.1016/j. advwatres.2005.08.006.

Loague, K., Heppner, C.S., Ebel, B.A., VanderKwaak, J.E.. The quixotic search for a comprehensive understanding of hydrologic response at the surface: Horton, Dunne, Dunton, and the role of concept-development simulation. Hydrological Processes 2010;24(17):2499-2505. URL: http://onlinelibrary.wiley.com/doi/10.1002/hyp.7834/abstract. doi:10.1002/hyp.7834.

Loague, K.M.. Impact of rainfall and soil hydraulic property information on runoff predictions at the hillslope scale. Water Resources Research 1988;24(9):1501-1510. URL: http://onlinelibrary . wiley.com/doi/10.1029/WR024i009p01501/abstract. doi:10.1029/WR024i009p01501.

Mantoglou, A., Wilson, J.L.. The Turning Bands Method for simulation of random fields using line generation by a spectral method. Water Resources Research 1982;18(5):1379-1394. URL: http:// onlinelibrary.wiley.com/doi/10.1029/WR018i005p01379/abstract. doi:10.1029/WR018i005p01379.

Maxwell, R.M.. A terrain-following grid transform and preconditioner for parallel, large-scale, integrated hydrologic modeling. Advances in Water Resources 2013:53:109-117. URL: http://www . sciencedirect.com/science/article/pii/S0309170812002564. doi:10.1016/j.advwatres.2012.10. 001.

Maxwell, R.M., Kollet, S.J.. Quantifying the effects of three-dimensional subsurface heterogeneity on Hortonian runoff processes using a coupled numerical, stochastic approach. Advances in Wa- 
ter Resources 2008;31(5):807-817. URL: http://www.sciencedirect.com/science/article/pii/ S0309170808000201. doi:10.1016/j.advwatres.2008.01.020.

Maxwell, R.M., Putti, M., Meyerhoff, S., Delfs, J.O., Ferguson, I.M., Ivanov, V., Kim, J., Kolditz, O., Kollet, S.J., Kumar, M., Lopez, S., Niu, J., Paniconi, C., Park, Y.J., Phanikumar, M.S., Shen, C., Sudicky, E.A., Sulis, M.. Surface-subsurface model intercomparison: A first set of benchmark results to diagnose integrated hydrology and feedbacks. Water Resources Research 2014;50(2):15311549. URL: http://onlinelibrary.wiley.com/doi/10.1002/2013WR013725/abstract. doi:10.1002/ 2013 WR013725.

Mejia, J.M., Rodriguez-Iturbe, I.. On the synthesis of random field sampling from the spectrum: An application to the generation of hydrologic spatial processes. Water Resources Research 1974;10(4):705711. URL: http://onlinelibrary.wiley.com/doi/10.1029/WR010i004p00705/abstract. doi:10 . 1029/WR010i004p00705.

Meyerhoff, S.B., Maxwell, R.M.. Quantifying the effects of subsurface heterogeneity on hillslope runoff using a stochastic approach. Hydrogeology Journal 2011;19(8):1515-1530. URL: http://link . springer.com/article/10.1007/s10040-011-0753-y. doi:10.1007/s10040-011-0753-y.

Meyerhoff, S.B., Maxwell, R.M., Graham, W.D., Iii, J.L.W.. Improved hydrograph prediction through subsurface characterization: conditional stochastic hillslope simulations. Hydrogeology Journal 2014;22(6):1329-1343. URL: http://link. springer.com/article/10.1007/s10040-014-1112-6. doi:10.1007/s10040-014-1112-6.

Michaelides, K., Wilson, M.D.. Uncertainty in predicted runoff due to patterns of spatially variable infiltration. Water Resources Research 2007;43(2):W02415. URL: http://onlinelibrary . wiley .com/ doi/10.1029/2006WR005039/abstract. doi:10.1029/2006WR005039.

Séguis, L., Cappelaere, B., Peugeot, C., Vieux, B.. Impact on Sahelian runoff of stochastic and elevation-induced spatial distributions of soil parameters. Hydrological Processes 2002;16(2):313-332. URL: http://onlinelibrary.wiley.com/doi/10.1002/hyp.337/abstract. doi:10.1002/hyp.337.

Singh, V.P.. Effect of spatial and temporal variability in rainfall and watershed characteristics on stream flow hydrograph. Hydrological Processes 1997;11(12):1649-1669. URL: http: //onlinelibrary.wiley.com/doi/10.1002/(SICI) 1099-1085(19971015) $11: 12\langle 1649:$ :AID-HYP495> 3.0.CO;2-1/abstract. doi:10.1002/(SICI) 1099-1085(19971015) $11: 12<1649::$ AID-HYP495>3.0.CO; $2-1$.

Smith, R.E., Hebbert, R.H.B.. A Monte Carlo Analysis of the hydrologic effects of spatial variability of infiltration. Water Resources Research 1979;15(2):419-429. URL: http://onlinelibrary.wiley . com/doi/10.1029/WR015i002p00419/abstract. doi:10.1029/WR015i002p00419.

Sulis, M., Meyerhoff, S.B., Paniconi, C., Maxwell, R.M., Putti, M., Kollet, S.J.. A comparison of two physics-based numerical models for simulating surface watergroundwater interactions. Advances in Water Resources 2010;33(4):456-467. URL: http://www.sciencedirect.com/science/article/ pii/S0309170810000229. doi:10.1016/j.advwatres.2010.01.010.

Tompson, A.F.B., Ababou, R., Gelhar, L.W.. Implementation of the three-dimensional turning bands random field generator. Water Resources Research 1989;25(10):2227-2243. URL: http:// onlinelibrary.wiley.com/doi/10.1029/WR025i010p02227/abstract. doi:10.1029/WR025i010p02227.

Woolhiser, D.A., Smith, R.E., Giraldez, J.V.. Effects of Spatial Variability of Saturated Hydraulic Conductivity on Hortonian Overland Flow. Water Resources Research 1996;32(3):671-678. URL: http://onlinelibrary. wiley.com/doi/10.1029/95WR03108/abstract. doi:10.1029/95WR03108. 